\title{
Neuregelung der UVG-Tarifierung ab 2007
}

\author{
Profitieren Sie jetzt von günstigeren Prämien
}

\section{Aufhebung der Einheitstarifierung}

Bis Ende 2006 waren die Versicherer im Bereich der obligatorischen Unfallversicherung UVG in den meisten Fällen an einen Einheitstarif gebunden. Dieser basierte auf einer gemeinsam erhobenen Risikostatistik und wurde vom Schweizerischen Versicherungsverband SVV als Empfehlung herausgegeben. Durch den Liberalisierungstrend in der Versicherungswirtschaft und durch die Revision des Kartellgesetzes wird der SVV künftig keine Empfehlungen mehr veröffentlichen. Dies führt dazu, dass die Versicherungsgesellschaften individuelle Prämiensätze einführen werden. Leider machen davon heute die wenigsten Versicherungsgesellschaften Gebrauch. So basieren die aktuellen Angebote meist noch auf der Empfehlung des Jahres 2006.

\section{FMH Insurance Services-Rahmenvertrag}

In Zusammenarbeit mit dem Versicherer KPT konnten wir für Sie in unserem Rahmenvertrag günstige Prämiensätze aushandeln. Profitieren Sie und Ihre Mitarbeiterinnen und Mitarbeiter von diesen ausgezeichneten Konditionen und wechseln Sie Ihre obligatorische Unfallversicherung nach UVG auf den nächstmöglichen Termin in den FMH Insurance Services-Rahmenvertrag. Bestellen Sie deshalb heute noch eine persönliche Offerte für Ihre Unternehmung.

\section{Profitieren Sie von unserem Angebot}

Ihre Bedürfnisse stehen bei uns im Mittelpunkt. Mit unserem Namen garantieren wir Ihnen die Betreuung durch professionelle Berater mit langjähriger Erfahrung im Versicherungs- und Bankenbereich. Dank unserer Unabhängigkeit können wir Ihnen das für Sie am besten geeignete Produkt anbieten.

\section{Antworttalon}

Vorname / Name

Adresse

PLZ / Ort

Geburtsdatum

Telefon Privat/Geschäft

Beste Zeit für einen Anruf

Bitte senden Sie mir eine UVG-Offerte des FMH Insurance Services-Rahmenvertrages. (Bitte legen Sie uns eine Kopie Ihrer aktuellen Versicherungspolice bei.)

Ich wünsche eine persönliche Beratung. Bitte rufen Sie mich an.

Ich interessiere mich für:

\begin{tabular}{ll} 
Krankenkasse & $\bigcirc$ Anlagen mit Kapitalschutz \\
Säule $3 a$ & $\bigcirc$ Rechtsschutzversicherung \\
$\bigcirc$ Pensionskasse BVG & $\bigcirc$ Berufshaftpflichtversicherung \\
\hline
\end{tabular}

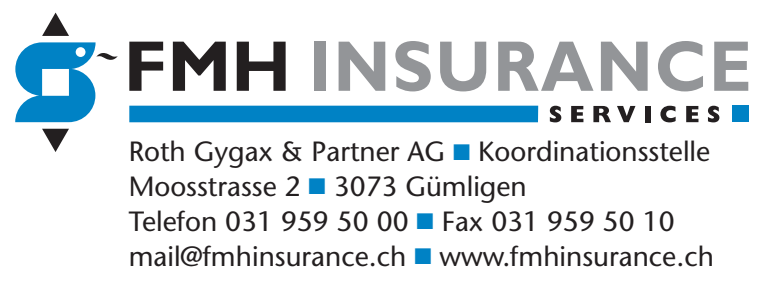

